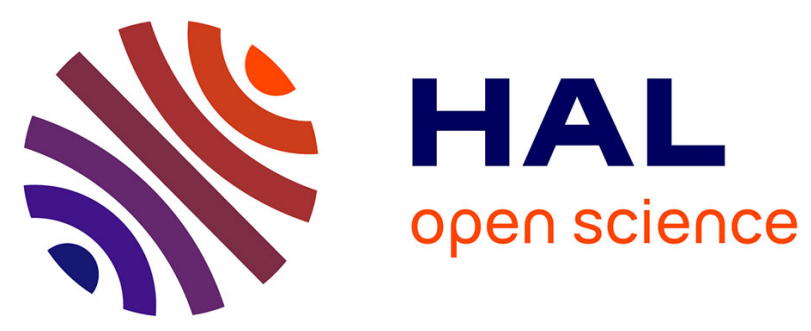

\title{
Mechanisms of torsade de pointes tachycardia in patients with spontaneous high-degree atrioventricular block: A modern look at old data
}

Guy Fontaine, Guoliang Li, Ardan Saguner, Robert Frank

\section{- To cite this version:}

Guy Fontaine, Guoliang Li, Ardan Saguner, Robert Frank. Mechanisms of torsade de pointes tachycardia in patients with spontaneous high-degree atrioventricular block: A modern look at old data. Journal of Electrocardiology, 2019, 56, pp.55-63. 10.1016/j.jelectrocard.2019.05.007 . hal-02967091

\section{HAL Id: hal-02967091 \\ https: / hal.sorbonne-universite.fr/hal-02967091}

Submitted on 14 Oct 2020

HAL is a multi-disciplinary open access archive for the deposit and dissemination of scientific research documents, whether they are published or not. The documents may come from teaching and research institutions in France or abroad, or from public or private research centers.
L'archive ouverte pluridisciplinaire HAL, est destinée au dépôt et à la diffusion de documents scientifiques de niveau recherche, publiés ou non, émanant des établissements d'enseignement et de recherche français ou étrangers, des laboratoires publics ou privés. 


\title{
Manuscript Details
}

\section{Manuscript number}

Title
JECG_2019_157

Mechanisms of torsade de pointes tachycardia in patients with spontaneous high-degree AV block: A modern look at old data

\section{Article type}

Research Paper

\begin{abstract}
Introduction: The mechanisms of torsade de pointes tachycardia (TdP) are incompletely understood. In this study, we aimed to illustrate to mechanisms of torsade de pointes tachycardia in patients with spontaneous high-degree AV block. Methods: This retrospective study reviewed old TdP recordings after ventricular temporary pacing interruption in 16 patients with spontaneous high-degree AV block (AVB). Five of them had also bipolar endocardial right ventricular (RV) apex recordings. Results: The QT interval during AVB at a mean heart rate of $38.9 \pm 7.5 \mathrm{bpm}$ was $653 \pm 67.2 \mathrm{~ms}$. The critical coupling interval $(\mathrm{CCl})$ between the last escape QRS during AVB and the first premature ventricular complex (PVC) was significantly shorter before the onset of TdP than before single PVCs and couplets. A morphologic crescendo of the escape T wave was observed before the onset of TdP, followed by a rhythmic and morphologic crescendo of PVCs. The escape RV apex electrograms showed the constant pattern of a rapid deflection similar to a Purkinje potential 40 to $80 \mathrm{~ms}$ after the onset of the QRS, superimposed on a smooth low amplitude signal in 4 of them. Conclusions: This suggests a local fast conducting fiber among electrically depressed apical myocardium. The major endocardial T wave prolongation before the earlier initiating PVC suggests a phase 2 reentry mechanism due to early after depolarization. The induced TdP can be due to the changing outputs from one or two simultaneous RV moving reentry circuits between depressed fibers and fast conducting ones, possibly located in the thin crista supraventricularis structure which has several connections with the septum and the RV free wall.
\end{abstract}

Taxonomy

Corresponding Author

Corresponding Author's Institution

Order of Authors

Suggested reviewers
Additional ECG Leads, Waveform Morphology, Adult

Guoliang Li

First Affiliated Hospital of Xi'an Jiaotong University

Guy Fontaine, Guoliang Li, Ardan Saguner, Frank Robert

Frank Marcus, Tong Liu, Aifeng Zhang

\section{Submission Files Included in this PDF}

\section{File Name [File Type]}

TdP cover letter-2019-3-27.docx [Cover Letter]

TdP-MS-submit-2019-3-27.docx [Manuscript File]

Figure 1.tif [Figure]

Figure 2.tif [Figure]

Figure 3.tif [Figure]

Figure 4.tif [Figure]

To view all the submission files, including those not included in the PDF, click on the manuscript title on your EVISE Homepage, then click 'Download zip file'. 


\section{COVER LETTER}

Dear Prof. Adrian Baranchuk, Editor-in-Chief, Journal of Electrocardiology

It is our great privilege to submit this original manuscript entitled "The mechanism of torsade de pointes tachycardia in patients with spontaneous high-degree AV block: A modern look at old data" to Journal of Electrocardiology.

Prof. Fontaine passed away last March, and left an unfinished text about his vision of torsade de pointes (TdP) that we completed. He was among the pioneers of modern electrophysiology, contributing to the understanding of bradycardia and tachycardia during the last 50 years We thought it could be a way to honor his contributions to our domain, his often original ideas, for instance in this paper, which is also a revue on long coupling torsade de pointes.

He retrieved 50 years old tracings, including 5 endocardial ones, in 16 patients without apparent cardiomyopathy besides a high-degree AV block, and reexamined them at the light of actual knowledge. It already showed TdP initiation via an increased dispersion of repolarization in adjacent ventricular myocardial fibers of patients with high degree AVB. Data are in keeping with phase 2 reentry as the triggering factors for TdP, and dual ventricular activation during TdP. He proposed the hypothesis that the RV crista terminalis, with its thin structure and wide surface and multiple connections could be the site for moving reentries with changing exits.

We confirm the manuscript is not under simultaneous consideration elsewhere. This study is an extension of previous data (ref 16-18), and substantially adds to our previous findings. All authors are responsible for the contents and have read and approved the final version of the manuscript for submission toEuropace.

Thanks in advance for your consideration,

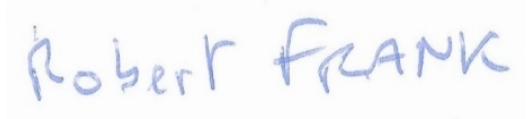

Dr Robert FRANK

robertfrank@sfr.fr, Institut de Cardiologie, Unité de Rythmologie, Hôpital de la Salpêtrière, 47-83 boulevard de I'Hôpital, 75651, Paris, France. Phone: + 336090782 94; Fax: 3342163057 
1, Institut de Cardiologie, Unité de Rythmologie, Hôpital Universitaire La Pitié-Salpêtrière, 47-83 boulevard de l'Hôpital, 75651, Paris, France.

2, Arrhythmia Unit, Department of Cardiovascular Medicine, First Affiliated Hospital of Xi'an Jiaotong University, No. 277 Yanta West Road, Xi'an 710061, China

3, Department of Cardiology, University Heart Center Zurich, Rämistrasse 100, 8091 Zurich,

Correspondence to:

11 Frank Robert (robertfrank@sfr.fr ), Institut de Cardiologie, Unité de Rythmologie, Hôpital de 12 la Salpêtrière, 47-83 boulevard de l'Hôpital, 75651, Paris, France.

13 Phone: +33609078294

$14 \quad$ Fax: 33-42163057

$15{ }^{*}$ The first two authors and last two authors contributed equally to this article and are shared 16 first authors and shared last authors, respectively. 


\section{Abstract}

Introduction: The mechanisms of torsade de pointes tachycardia (TdP) are incompletely understood. We aimed to illustrate to mechanisms of torsade de pointes tachycardia in patients with spontaneous high-degree AV block.

Methods and results: This retrospective study reviewed old TdP recordings after ventricular temporary pacing interruption in 16 patients with spontaneous high-degree AV block (AVB). Five of them had also bipolar endocardial right ventricular (RV) apex recordings. The QT interval during AVB at a mean heart rate of $38.9 \pm 7.5 \mathrm{bpm}$ was $653 \pm 67.2 \mathrm{~ms}$. The critical coupling interval (CCI) between the last escape QRS during AVB and the first premature ventricular complex (PVC) was significantly shorter before the onset of TdP than before single PVCs and couplets. A morphologic crescendo of the escape T wave was observed before the onset of TdP, followed by a rhythmic and morphologic crescendo of PVCs. The escape RV apex electrograms showed the constant pattern of a rapid deflection similar to a Purkinje potential 40 to $80 \mathrm{~ms}$ after the onset of the QRS, superimposed on a smooth low amplitude signal in 4 of them.

Conclusions: The major endocardial T wave prolongation before the earlier initiating PVC suggests a phase 2 reentry mechanism due to early after depolarization. The induced TdP can be due to the changing outputs from one or two simultaneous RV moving reentry circuits between depressed fibers and fast conducting ones, possibly located in the thin crista supraventricularis structure which has several connections with the septum and the RV free wall.

Key words: torsade de pointes; electrocardiogram; endocardial mapping; atrioventricular block. 
Torsade de pointes tachycardia (TdP) a rare but important cause of sudden death in young people with the genetically determined long QT syndrome. In these patients, the length of the QT interval is directly related to the arrhythmic risk modulated by genetic factors. TdP is also a major concern of the pharmaceutical industry for the development of cardiac and non-cardiac drugs. It is also important to understand the basic mechanisms of this form of polymorphic ventricular tachycardia (VT) when it occurs in patients with high-degree atrioventricular (AV) block. In 1966, this arrhythmia received the descriptive term of "Torsade de pointes". This name was chosen to describe the QRS that twists around the isoelectric line by Desertenne.[1] Scientists from Germany reproduced this ECG pattern experimentally by pacing both ventricles in isolated pig hearts and confirmed the original hypothesis of two alternating activation sites[2]. Moreover, cellular electrophysiologists.[3, 4] determined that early after depolarization (EAD) should be the initial fundamental mechanism, as EAD are bradycardia-dependent and favored by QT prolongation, as are TdP. However, their perpetuation mechanism remains still discussed between automaticity and reentry. The role of $M$ cells remains also discussed with diverging data from myocardium wedge preparations and from whole intact hearts.[5]

The ECG recordings presented in this manuscript were performed between 1964 and 1970 , at a time when most of these data was unknown. Some patients with coronary artery disease had "transient ventricular fibrillation (VF)", a term associated with poor prognosis, and others with complete AV block had what was called "tachyrhythmia" in English publications.[6] There was also a waiting period with temporary pacing leads in those early times of pacemaker (PM) implantation. These original studies were undertaken to demonstrate that the arrhythmia could be prevented by pacing in some of these patients and that it reoccurred when pacing was interrupted. When this was confirmed, permanent 
64 pacemaker implantation became a routine procedure, but the exact mechanism of TdP and their relation to VT and VF was not fully elucidated. In this study, we aimed to synthesize the actual knowledge with the data from these old observations. We also propose a potential site for the localization of this type of TdP.

\section{Methods}

Definitions

TdP has a complex definition, and what is specific is not really the torsade of QRS by itself, as

there are other types of polymorphic VT, but its initiating complex, with a long short sequence on a long QT. In this series of AVB induced long QT, aborted sequences could be limited to the initial PVC or followed by one or several cycles of non-sustained VT with the same or different morphologies, with abrupt or progressive morphological changes, and sometimes degenerating into VF.

\section{Study population}

The study population consisted of 16 patients exhibiting 107 typical episodes of TdP during spontaneous high-degree AV block, and studied by surface ECG recordings 50 years ago. As explained above, these patients were waiting for definite PM implantation, PM replacement, and therefore had a temporary pacing bipolar electrode connected to an external pacemaker. They were $>65$ years old (range 66-80 years), thirteen women and three men, and none was taking drugs known to prolong the QT interval. All had normal serum laboratory tests including serum potassium levels. There were no data on any familial disease. They all presented with type 2 second degree (Mobitz II) or third degree AVB. Most of them had well tolerated short episodes of TdP (less than $5 \mathrm{sec}$ ) preceded by premature ventricular 
complexes (PVCs), couplets, triplets etc. After the recording of several episodes on a standard ECG recorder, it was noticed that some of them had the same QRS morphologies at the initiation of the TdP. Therefore, to gain further insight, long-term bipolar ECGs were tape recorded in all, between a negative skin electrode placed in position V1 and a positive one placed in position V4, similar as lead II orientation, but in front of the right ventricle and the septum. Five of them had a simultaneous endocardial recording with the $1.2 \mathrm{~cm}$ bipolar pacing endocardial electrode. The precordial as the endocardial electrodes were connected to Tektronix $3 A 9$ differential amplifiers with a band pass of DC to $60 \mathrm{~Hz}$, in those times when specific electrophysiological equipment was not available. The amplifier output was connected to a tape recorder (Ampex SP 300, Redwood City, CA, USA). The tapes were subsequently reviewed with an oscilloscope (Tektronix 561A-3A72) to select episodes of TdP, which were printed on a channel ECG machine (Sanborn EH101371 Cambridge, MA, USA), later on a dual channel industrial ink pressure recorder (Clevite Brush 220 Chart Recorder Cleveland, OH, USA). All data were collected and reviewed by one of us (GHF) before they were entered into a spreadsheet for statistical analysis (Dell Statisticav12, Tulsa, OK, USA). The critical coupling interval ( $\mathrm{CCl}$ ) between the last beat during $\mathrm{AVB}$ and before the first abnormal ventricular events such as PVCs, couplets, triplets and TdPs (defined by $>3$ consecutive complexes) were identified, numbered and measured with a caliper with a precision $<20 \mathrm{~ms}$. Finally, the occurrence of TdP after pacing interruption was repeatedly assessed. The CCls and the amplitude of the last TdP beats were also analyzed. Histograms of each numeric variable were drawn to identify outliers. The comparison of populations with continuous variables was performed by the Student's test. A p value $<0.05$ was considered significant. 
Preliminary results of this study have been previously published.[7-9] This study is a more detailed and extended analysis of these previous reports.

\section{Results}

The QT interval during AV block at a mean heart rate of $38.9 \pm 7.5 \mathrm{bpm}$ was $653 \pm 67.2 \mathrm{~ms}$

Ventricular event timings

All ventricular arrhythmic events were preceded by long diastoles from 1000 to more than 1500 ms terminated by a junctional or ventricular escape (Figure 1A). After this escape beat, PVC occurred late, after the end of the prolonged T wave. Single PVCs were observed in 160 episodes with a CCl of $672 \pm 44 \mathrm{~ms}(550-760), 121$ couplets with a CCl of $676 \pm 37 \mathrm{~ms}$ (560760), 21 triplets with a CCl of $644 \pm 39 \mathrm{~ms}(560-720)$, and TdP in 107 episodes with a $\mathrm{CCl}$ of $639 \pm 52 \mathrm{~ms}(500-760)(\mathrm{p}<0.05$ for TdP vs. single PVCs and couplets), suggesting a trend to shorter coupling intervals for the onset of sustained TdP. The case presented in Figure 1A was the only one in which TdP did degenerate into fast regular VT and then VF. In the five patients with endocardial recordings (Figure 1B), the onset of endocardial activation on the RV apex diagnostic catheter was observed prior to or at the same time as the initiating PVC. TdP occurred separated by long periods without PVC in 11 patients.

Repolarization crescendo

The severe bradycardia induced progressive modifications of the T wave durations and shapes of the escape rhythms reflect an increasing dispersion of repolarization (Figure $1 \mathrm{~A}, 2 \mathrm{~A}$ and $2 \mathrm{C}$ ) during several seconds before the first PVC. This "morphologic crescendo" announcing the onset of TdP was observed in 12 patients (75\%), sometimes associated with monstrous "bumps" better seen on the endocardial recordings, bumps which could also progressively regress (Figure 2B), with PVC's disappearance. 
Rhythmic crescendo

TdP episodes were generally preceded by a "rhythmic crescendo" of isolated PVCs,

then couplets, triplets, quadruplets etc. (Figure $1 \mathrm{~A}$ and $2 \mathrm{~A}$ ) inducing the classical long-short initiating cycle sequence. This was observed in all 16 cases. A decrescendo of these arrhythmias was observed in 11 patients after the end of a TdP period.

Ventricular complex morphologies

Periods of non-sustained TdP (up to around 30 seconds) showed several repetitive, almost identical morphologies, which could be classified into four major categories: 4 patients had a single one, 5 patients had 2, 6 patients had 3, and one had 4 morphologies (Figure 2C) with fusion beats. Of these, the initiating PVC morphology could be negative or positive in the bipolar precordial ECG recordings in the same patient (Figure $2 \mathrm{C}$ and $2 \mathrm{D}$ ). An abrupt change of QRS orientation from upward to downward is illustrated in Figure 3A, at the end of the TdP and was observed in four cases, the others without major change from the preceding cycles. The single bipolar precordial derivation from the tape recordings did not allow a clearer identification of the site of PVC origin than a leftward and descending activation for a positive wave, which could be originating from the RV outflow tract (RVOT), and a negative one from the inferior part of the ventricles (Figure 2C), and fusions complexes.

Endocardial data were available from 5 patients, all from the RV apex bipolar electrode, and detailed elsewhere.[8, 9] All had an escape electrogram (EGM) with a sharp Purkinje-like potential synchronous with the middle of the QRS, superimposed to a low voltage surrounding signal in 4 of them (Figure 1B). A similar potential could be observed at the onset of the initiating PVC in case 1 from Figure 1B), preceded by a smooth potential before the QRS 
onset in continuity with the T wave of the escape beat, interpreted as EAD's from the same region. This kind of thin potential was observed at other moments of the recording with a later timing, during PVC's of other morphologies (Figure 2D) in the same patient. Fragmentations were recorded in the next beats suggesting local inhomogeneity of the activation process (Figure 1B and 2D). On the nowadays retrieved tracings, complete endocardial recordings during TdP were only available for one patient (Figure 3B).

\section{Discussion}

Since the original description of TdP in complete AV block,[1] the electrocardiographic predictors of this situation are still discussed. Many previous work made possible to explain some of the observations made in our old ECG recordings. They gave further insight into the mechanisms of initiation and perpetuation of TdP in high-degree AV block. We are potentially dealing with a functional arrhythmogenic substrate, reflected by EGM fragmentations[9] which are not due to a fixed anatomic obstacle such as fibrosis resulting from the sequel of myocardial infarction, but likely to anisotropic propagation creating functional blocks. A relation with the familial forms of LQTS has also been proposed, as not all patients with chronic AV block develop long QT and TdP. There is genetic predisposition.[10]

\section{The initiating complex}

TdP were reproducibly observed in all patients. After interruption of ventricular pacing there is a junctional escape rhythm with severe bradycardia and progressive $\mathrm{T}$ wave prolongation and shape modifications (Figure 1B and 2). They reflect an increase in the physiologic heterogeneity of action potential durations.[11] After an abrupt change of the cycle length these modifications are progressive, but also differ between myocardial and 
Purkinje fibers.[12] This progression explains the rhythmic as well as the morphologic crescendo with irregular huge $T$ wave bumps (Figure $1 \mathrm{~A}$ and $2 \mathrm{~A}$ ), announcing the occurrence of TdP. This increased repolarization duration can induce a prolonged repolarization dependent re-excitation, early afterdepolarisations, a phase 2 reentry marked as above by bumps on the prolonged action potentials descending part. These potentials can activate excitable neighboring myocardium, giving rise to the initiating PVC. It has been also demonstrated in patients, in familial TdP as in chronic AVB by RV endocardial map recordings.[13-15] It was also recognized on our bipolar recordings at the RV apex with wide wave (Figure 2A) before the initiating PVC. The initiating role of subendocardial Purkinje fibers idiopathic VF. This was probable in one of our cases (Figure 2D).

The following QRS'S

There is still a controversial debate about the nature of those following PVC's, new phase 2 potentials progressively shorten with further shorter cardiac cycles, phase 2 reentry should stop after some of them. Perpetuation should be due to another mechanism. Conversely the prematurity of the triggering PVC also enhances the repolarizations disparities, thus demonstrated in Allessie's leading circle model in a thin myocardial preparation without fibrosis.[16] Its moving location can explain the QRS changes, many times reproduced in experimental myocardial preparations as in full heart models, and interpreted as a rotor moving within the ventricles. This is consistent from El Sherif's experimental acute dog model 
with AV block in 1997, and Vandersickel et al[17] in a chronic one, with TdP induced after one or several EAD's. They show reentry around zones of functional blocks, with changes in circuit locations explaining the morphological changes. This is the most probable mechanism in the majority of experimental setups in AV block with drug induced TDP. It seems difficult to demonstrate it in humans, as it had been for rotors in ventricular fibrillation. However, the coexistence of fast conducting fibers with depressed ones suggested by our endocardial recordings are consistent with a substrate for localized reentry.

In our series some patients had sometimes only one morphology after the initiating complex, but the others had at least two main upward and downward orientations (Figure 2C, $2 \mathrm{D}$ and $3 \mathrm{~A}$ ) with fusion beats in between. For instance, in Figure 3B, once the TdP initiated, our RV apex endocardial bipolar signal changed cycle after cycle, with small modifications on syndrome with two accessory pathways in atrial fibrillation (Figure 3C). 
TdP may stop spontaneously after any form of intramyocardial block, as presented in Figure $3 \mathrm{~A}$ and $3 \mathrm{~B}$, or when there is less dispersion of repolarization visible on the surface tracing as shown on Figure $2 \mathrm{~B}$, or when reestablishment of pacing prematurely invades the myocardium in the opposite direction precluding reentry (Figure 3C).

\section{Site of origin}

The site of origin of the initiating beat has been studied by Birati et al[18] from surface ECG recordings in a population of 50 patients with various TdP etiologies, familial, drug induced or AV block. They found no correlation between etiology and QRS morphologies. From 874 impending episodes, 70\% had initial PVC morphologies suggesting an RVOT site, 13\% from the RV inferior wall, and 17\% from the LV inferior wall. Limited to the 216 episodes with 12 lead ECG recordings, 48\% were RVOT, 41\% LV inferior wall, 11\% RV inferior wall. 151 of those episodes were followed by TdP, of which $56 \%$ had an initial PVC compatible with an RVOT site, and the others from LV (32\%) or RV (13\%) inferior walls. Despite the limitations of QRS analysis, it shows that most of the initiating complexes came from the RV. It fits with the early occurrence of the RV apical endocardial potentials in our 5 patients as shown in Figure 1B. It was also the site of EAD recordings in patients with familial LQTS.[14, 15] To our knowledge, there has been no other endocardial recording of an RV initiating PVC in AVB related TdP than in our 5 patients. However, as the patients without EGM recording had the same morphologies on their bipolar precordial ECG, we can assume that they could also have a RV origin. 
It is interesting to speculate that if a thin structure exists in the ventricles, apt to propagate a circular activation front, as in Allessie's model, it should be in the right one, which has a thickness of $2-3 \mathrm{~mm}$ as opposed to 5 to $9 \mathrm{~mm}$ for the left one. GHF speculated that the region of interest for TdP to propagate could be the crista supraventricularis. In the anatomic study of two hearts from two patients (Figure 4 A, B and C), the crista has a thickness of one to three millimeters, as Thomas James published in 1985,[19] and can get thinner on its course.[20] Therefore, to the best of our knowledge, we are the first to propose that in the human heart, the crista supraventricularis, the thinnest part of the RV, could be the anatomical substrate for most of those TdP. The crista supraventricularis is described by T. James as a U-shaped folded structure connected to the high septal area, and with fibers diverging as a fan to the anterior RV. He stated that the crista "is crucially located to join the interventricular septum and left ventricle to much of the RV free wall".[19] This structure can be linked to the interventricular septal Purkinje net and could transmit activation to the antero-superior and postero-inferior sulci. In that structure the genesis of the QRS changing from upward to downward activation morphologies with fusion could be (Figure 4D) two simultaneous circuits with slightly different cycle lenghts and entrance block from one another, as suggested in our Figure 3A. Another mechanism could be one moving circuit with slight cycle length changes and dual changing exits, with Wenckebach type conduction block to the rest of the ventricle (Figure 4D). This second hypothesis could explain the abrupt changes in two opposite directions observed at the beginning and at the end of a TdP (Figure $3 \mathrm{~A})$, the two exit sites being then able to activate one after the other. least with the opportunity of the rare cases with ablation of PVC's initiating TdP. 
279 in our hospital before 1970. The tape recordings are no more available. Only a single precordial bipolar ECG lead, in front of the RV and the septal area, was recorded in most of

281 the tracings. Therefore, some interesting information may have been missed. Endocardial 282 recordings only explored the RV apex, and there is no data on other locations, as recordings 283 from the crista supraventricularis and from the left ventricle.

\section{Conflict of interest}

285 Authors have no conflict of interest to disclose.

\section{Funding}

287 This research did not receive any specific grant from funding agencies in the public, 288 commercial, or not-for-profit sectors

\section{Ethics}

290 This is a retrospective study. When Guy H. Fontaine carried out these studies between 1965 291 and 1970, there were no ethical standards of a responsible committee on human 292 experimentation (institutional or regional) as established 10 years later. This investigation was 293 approved by the chief of cardiology as there was a clinical need for these patients to define 294 in whom PM implant would be useful. An oral approved consent by the patient was obtained 295 before the procedure. 
298 We extend our gratitude to Frank I. Marcus, MD for his collaboration in the editing of the 299 manuscript and Yvette Wingfield for her secretarial assistance, The University of Arizona 300 College of Medicine, Tucson, AZ, USA. 


\section{References:}

302 [1] Dessertenne F. [Ventricular tachycardia with 2 variable opposing foci]. Archives des maladies du coeur et des vaisseaux. 1966;59:263-72.

304

[2] D'Alnoncourt CN, Zierhut W, Blüderitz B. "Torsade de pointes" tachycardia. Re-entry or

305 focal activity? British heart journal. 1982;48:213-6.

306 [3] Brachmann J, Scherlag BJ, Rosenshtraukh LV, Lazzara R. Bradycardia-dependent triggered 307 activity: relevance to drug-induced multiform ventricular tachycardia. Circulation. $1983 ; 68: 846-56$

309 [4] Damiano BP, Rosen MR. Effects of pacing on triggered activity induced by early 310 afterdepolarizations. Circulation. 1984;69:1013-25.

311 [5] Kohl P, Bollensdorff C, Quinn TA. Resolving the M-cell debate: mechanics matters. Heart rhythm. 2011;8:e1; author reply e2.

[6] Jensen G, Sigurd B, Sandoe E. Adams-Stokes seizures due to ventricular

tachydysrhythmias in patients with heart block: prevalence and problems of management. Chest. 1975;67:43-8.

316 [7] Fontaine G. A New Look at Torsades de Pointesa. Annals of the New York Academy of 317 Sciences. 1992;644:157-77.

318 [8] Li G, Saguner AM, Akdis D, Fontaine GH. Intramyocardial block in patients with 319 atrioventricular block. Journal of investigative medicine : the official publication of the 320 American Federation for Clinical Research. 2018;66:1-4.

321 [9] Li GL, Saguner AM, Fontaine GH, Frank R. Fragmented endocardial signals and early 322 afterdepolarizations during torsades de pointes tachycardia. Cardiology journal. 2018. 

in combination with aging causes SCN5A-linked hereditary Lenegre disease. Journal of the American College of Cardiology. 2003;41:643-52.

[11] Moe GK, Mendez C. Functional block in the intraventricular conduction system.

327 Circulation. 1971;43:949-54.

[12] Denker S, Shenasa M, Gilbert CJ, Akhtar M. Effects of abrupt changes in cycle length on refractoriness of the His-Purkinje system in man. Circulation. 1983;67:60-8.

[13] Bonatti V, Rolli A, Botti G. Recording of monophasic action potentials of the right ventricle in long QT syndromes complicated by severe ventricular arrhythmias. European heart journal. 1983;4:168-79.

[14] Zhou JT, Zheng LR, Liu WY. Role of early afterdepolarization in familial long QTU syndrome and torsade de pointes. Pacing and clinical electrophysiology : PACE. 1992;15:2164-8.

[15] Miwa S, Inoue T, Yokoyama M. Monophasic action potentials in patients with torsades de pointes. Japanese circulation journal. 1994;58:248-58.

[16] Allessie MA, Bonke FI, Schopman FJ. Circus movement in rabbit atrial muscle as a mechanism of tachycardia. III. The "leading circle" concept: a new model of circus movement in cardiac tissue without the involvement of an anatomical obstacle. Circulation research. 1977;41:9-18.

[17] Vandersickel N, Bossu A, De Neve J, Dunnink A, Meijborg VMF, van der Heyden MAG, et al. Short-Lasting Episodes of Torsade de Pointes in the Chronic Atrioventricular Block Dog Model Have a Focal Mechanism, While Longer-Lasting Episodes Are Maintained by Re-Entry. 
346 [18] Birati EY, Belhassen B, Bardai A, Wilde AA, Viskin S. The site of origin of torsade de 347 pointes. Heart. 2011;97:1650-4.

348 [19] James TN. Anatomy of the crista supraventricularis: its importance for understanding 349 right ventricular function, right ventricular infarction and related conditions. Journal of the 350 American College of Cardiology. 1985;6:1083-95.

351 [20] Kosinski A, Kozlowski D, Nowinski J, Lewicka E, Dabrowska-Kugacka A, Raczak G, et al. 352 Morphogenetic aspects of the septomarginal trabecula in the human heart. Archives of 353 medical science : AMS. 2010;6:733-43. 
Figure legends:

$357 \quad$ Figure 1

358 A, Surface ECG (single lead derived from bipolar chest leads between V1 and V4): interruption of ventricular pacing (first line) shows long diastoles due to high degree AV block leading to a rhythmic crescendo and finally stable rapid VT pattern degenerating into VF converted by 100 Joules monophasic DC shock. Skeletal muscle artifacts (asterisk) are related to convulsions during two seconds at the recovery of consciousness (paper speed $25 \mathrm{~mm} / \mathrm{s}$ ).

B, ECG and apex EGM of last escape complex and initiating PVC in the 5 patients with both tape recordings.

Top tracings: Surface ECG (single bipolar V1-V4 chest leads).

Bottom tracings: Synchronous bipolar RV apex EGM replayed from the magnetic tape. The escape endocardial potential had in each patient the same pattern of a fast sharp signal similar to a Purkinje potential superposed on a wider low amplitude signal in four of them, as a remote myocardial signal, or a depressed local myocardial signal. A similar sharp signal is visible after the initiating TdP PVC's onset as the transmission from a remote origin. It should be part of the apex Purkinje system.

\section{Figure 2}

374 A, ECG (bipolar precordial V1-V4 $25 \mathrm{~mm} / \mathrm{s}$ ) and below EGM repolarization crescendo (patient

3754 in Figure 1B recordings), before the PVC occurrence.

B, ECG repolarization decrescendo with PVC disappearance.

C, ECG (bipolar precordial V1-V4 25mm/s): ECG strips displaying the rhythmic crescendo with negative isolated PVC or salvos up to seven, suggesting a single circuit reentry. TdP are only induced by a positive one after a prolonged T wave. The progressive QRS changes in shape 
during the torsade on the three bottom strips suggest for each one a different site, and could be explained by a progressive fusion of two circuits with a small different phase or one with two exit points with different conduction times.

D, Four different TdP onsets ECG and EGM in patient 1 from Figure 1B. Note the different timings of the sharp Purkinje like signals within the different PVC's (arrows or stars).

Figure 3

A, Same legend as 2C: The end of TdP with longer intervals in the first line (asterisk) suggests a Wenckebach phenomenon on the last active circus movement (CM). An end of TdP without slowing is seen in the second line suggesting a Mobitz type 2 block on the last active CM (bold arrow). However, a small pacing spike before the last QRS could be subthreshold, but could also have interrupted the torsade as there is a paced ventriculogram after the end of the torsade. Abrupt change in the direction of QRS tips at the end of the third line (thin arrow) suggests a Mobitz type 2 block on only one of the two CMs.

B, Simultaneous recordings $(25 \mathrm{~mm} / \mathrm{s})$ of a TdP with the precordial bipolar V1-V4 ECG (upper trace and numbers) and its corresponding apex EGMs (lower trace and numbers) demonstrating two simultaneous ventricular activations moving cycle after cycle in the RV from the same patient. (Measures with a screen caliper calibrated with the ECG paper grid $1 \mathrm{~mm}=40 \mathrm{~ms})$.

On the surface ECG, after an initiating positive QRS on a huge T wave, next QRS and its following one have a strange shape and seem biphasic. The next QRS's are negative, slightly irregular, with a progressive narrowing until beat 11 . They then widen for the two last ones. These morphological changes suggest fusions from different activations waves, confirmed by the EGMs. 
The initiating apex EGM occurs at the onset of the surface QRS, and starts with a bipolar fast signal at the end of the prolonged T wave (circle), suggesting a local focal origin, followed by a remote, low voltage, $120 \mathrm{~ms} S$ wave. The next one starts with the early same fast signal, but the remote part of the signal changes, as the QRS shape, initiating the torsade for the next 11 beats. The fast signal is still seen within the third EGM, at the nadir of its $\mathrm{S}$ wave. A close analysis of next EGMs reveals two simultaneous cycle lengths, of 240 and $280 \mathrm{~ms}$. It is obvious by looking at EGM 8, which has several components, as its surroundings. The first, marked by an ascending arrow under the tracing, consist of an early negative small potential synchronous to the onset of the surface QRS with an immediate positive wave. The next EGMs have also an early negative initial biphasic wave of increasing amplitude, with an identical cycle of $280 \mathrm{~ms}$, and $260 \mathrm{~ms}$ for the last two QRS. When transposing the $280 \mathrm{~ms}$ intervals to prior EGMs, there are slurrings (arrows) on EGM S waves, still premature to the surface QRS, suggesting that this potential is fused with earlier EGMs end of other origin. The last component of EGM 8 is the second positive one, marked by descending arrows, and clearly seen on cycles 9 and 10, progressively fused with the first component, and may be deforming the $\mathrm{R}$ wave of the two last electrograms reflecting a last change of activation. This positive potential can be also recognized by reporting 240 ms on EGM 7 to 5 , and may be 4 and 3 . Both circuits seem to initiate simultaneously after EGM 3, as if there had been a bifurcation in the activation pathways.

C, Example of dual ventricular activation by two different ventricular inputs in a case of two accessory pathways in atrial fibrillation resembling TdP 
427 A, Human autopsy heart from a patient with coronary artery disease and chronic myocardial 428 infarction showing the crista supraventricularis of the right ventricle (two-sided arrow), the 429 thinnest structure in the human ventricles, in proximity to the His-Purkinje system. This crista may correspond to the region of adjacent fibers leading to a phase 2 reentry and initiation of

431 TdP in patients with high degree AV block. Arrow: Crista supraventricularis. Note left septo432 apical scar.

433 B, Autopsy heart from a human without cardiac disease displaying the location of the crista 434 supraventricularis (two-sided thin arrows). PA, white tissue in pulmonary artery; TV, tricuspid 435 valve. Tweezers clamp under the cordae tendinae of the TV septal valve.

436 C, The opening of the same heart along the crista displays the extreme thinness of this 437 structure as compared to the thicker right ventricular free wall (two-sided bold arrow). Thick 438 arrow: 3mm for RV free wall. Thin arrow: $1 \mathrm{~mm}$ for Crista supraventricularis

439 D, Two hypothesis for the TdP inside a cardiac structure. The reentrant mechanisms occur 440 between different fibers with different functional properties that could be defined as "Normal" 441 or "Depressed" (1): One moving circuit and two exit points (2), or two different simultaneous 442 circuits isolated one from the other (3). Since reentry can happen within a small area of myocardium two different circus movement tachycardia circuits can be simultaneously 444 present, yielding different morphologies and cycle lengths as illustrated in 3B. 


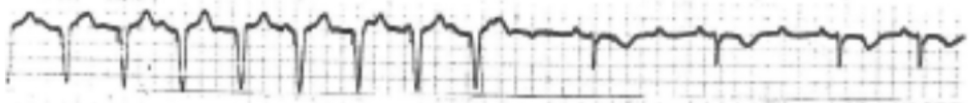

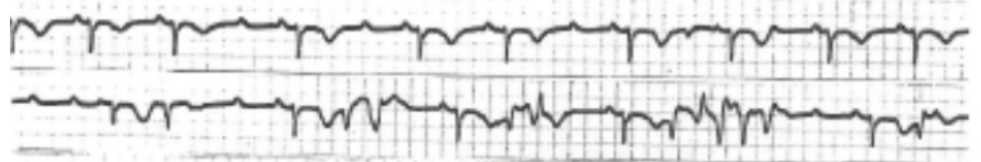

mar rampmompmpmp

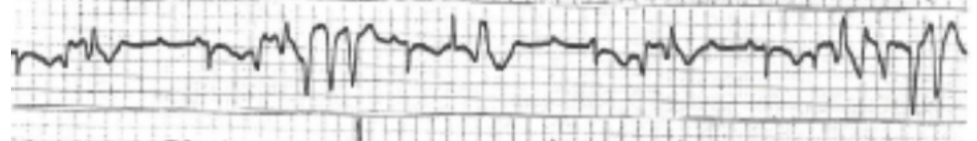

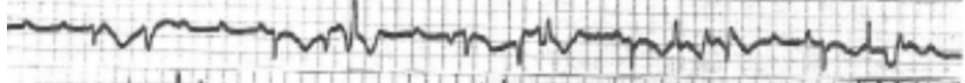

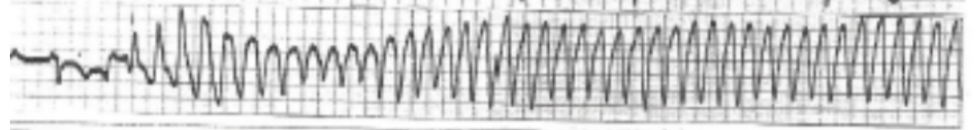

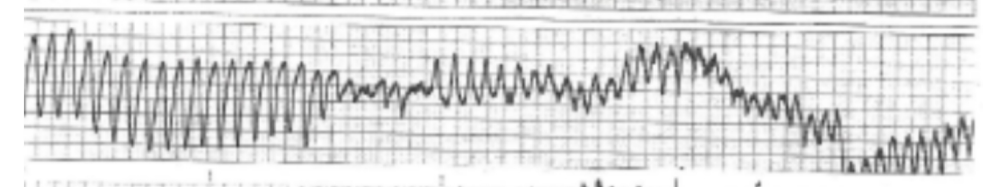

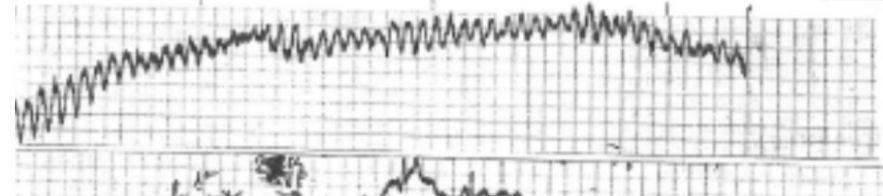

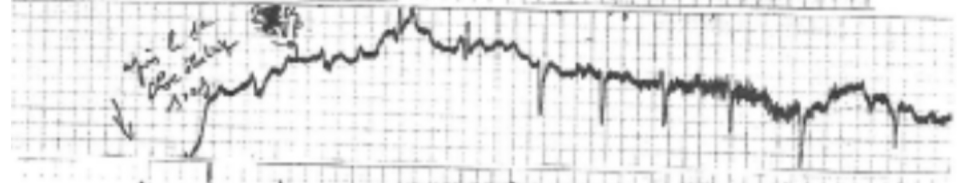

holy

A

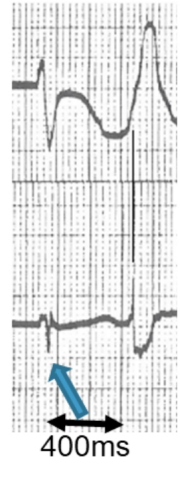

1

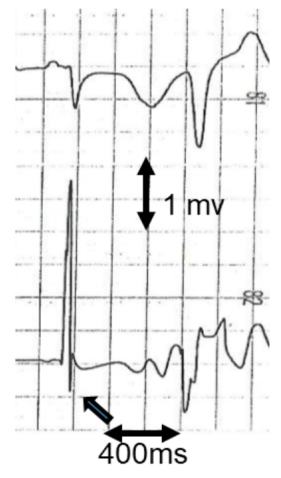

2

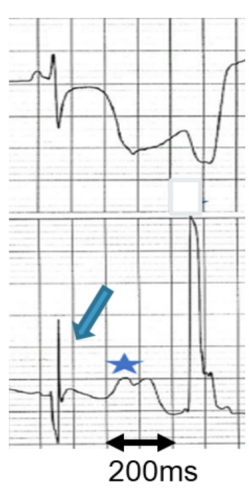

3

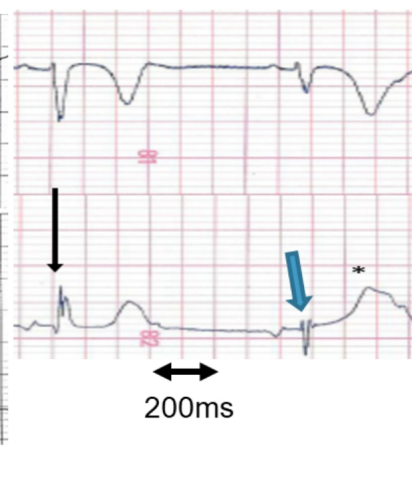

4

5

B 


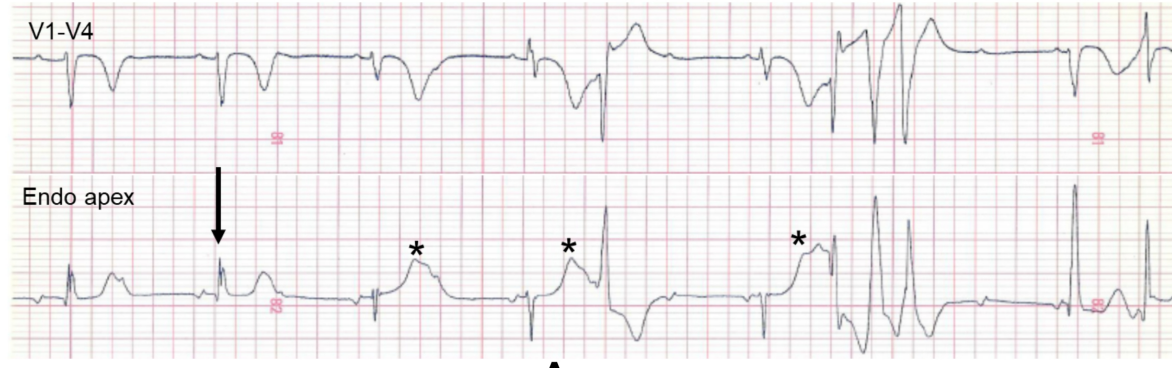

A
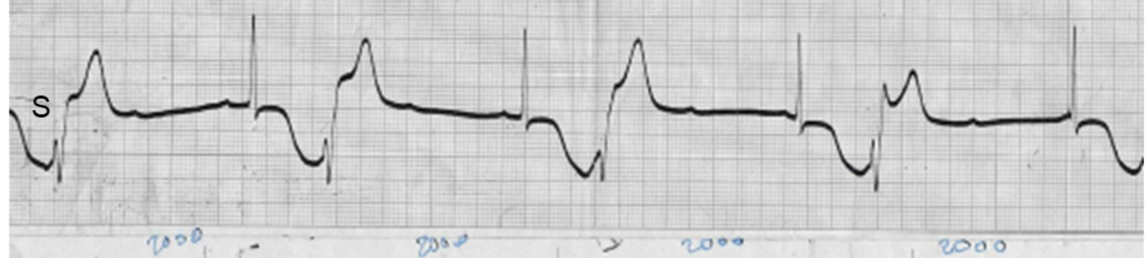

$32000+2000$

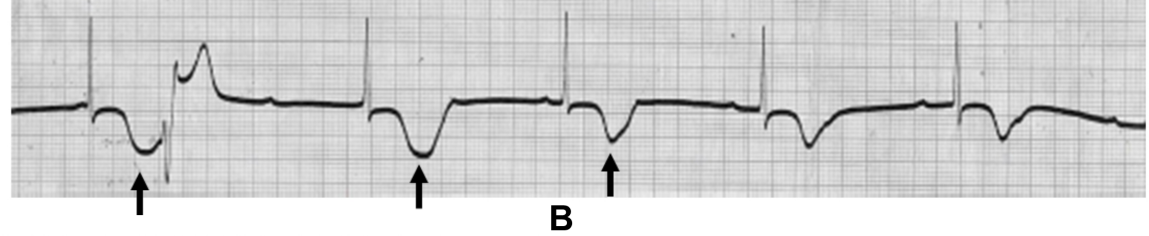

-

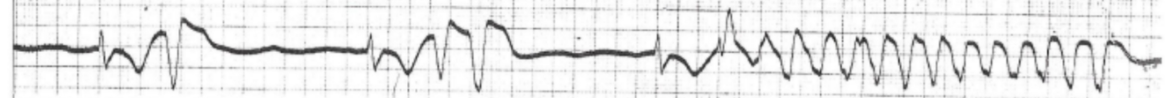

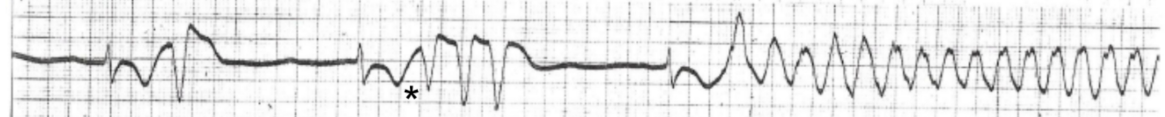

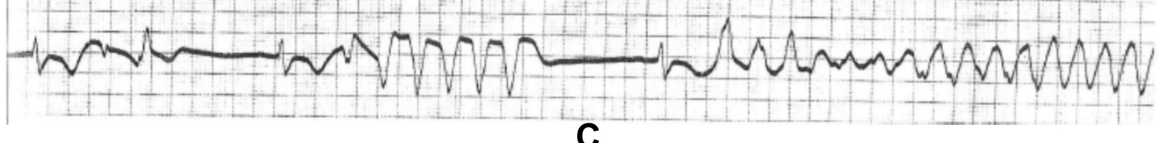

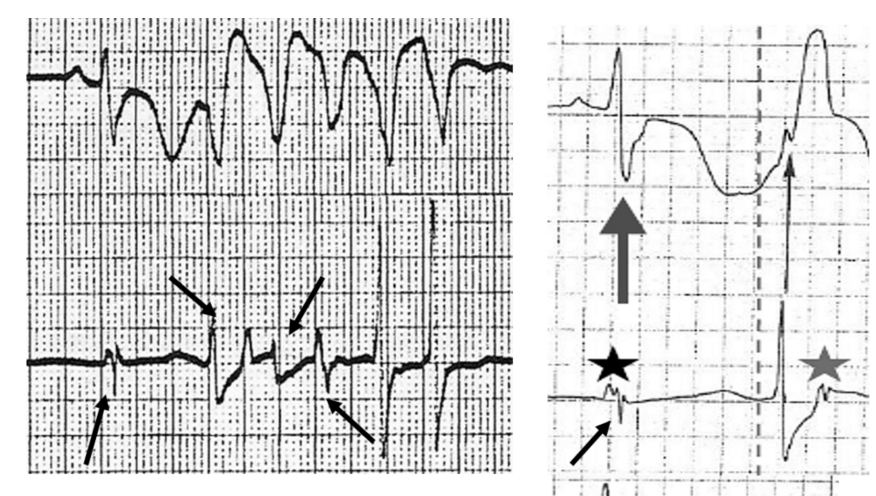

$25 \mathrm{~mm} / \mathrm{s}$
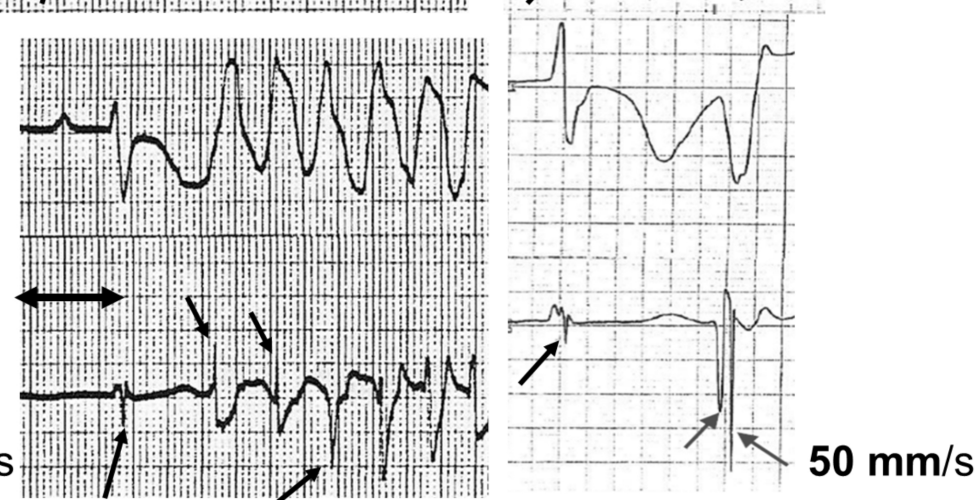

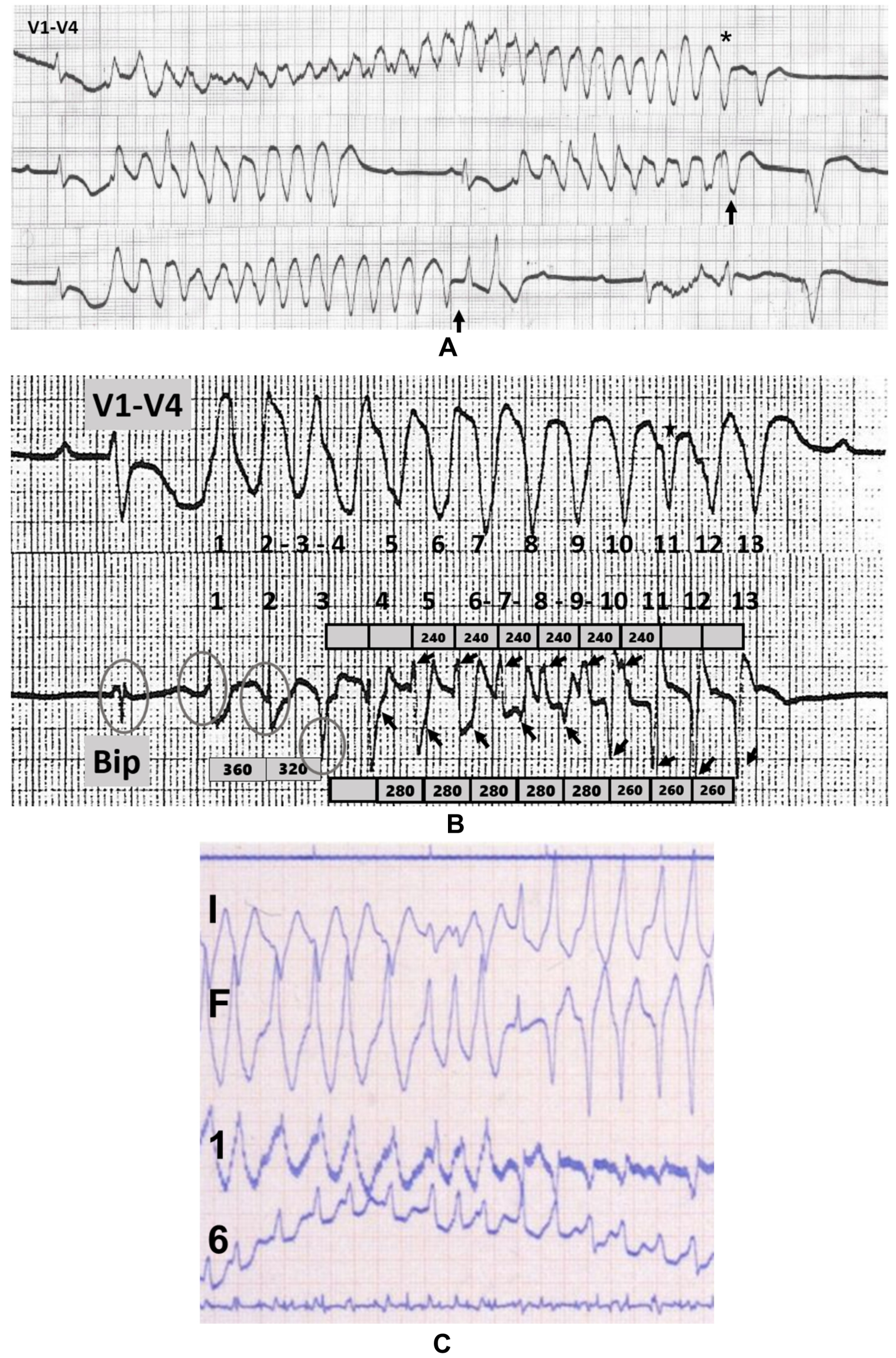

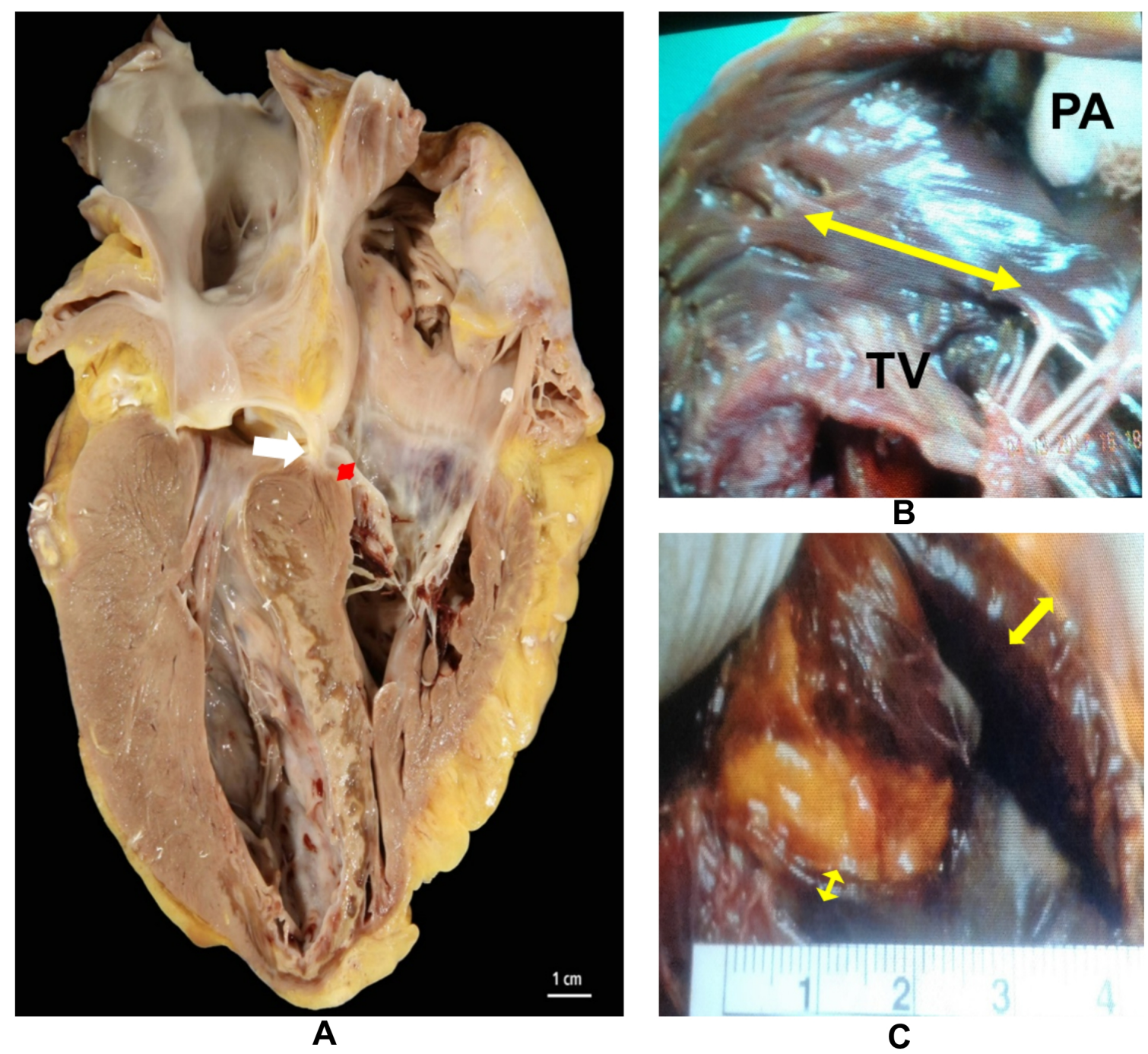

Exit a

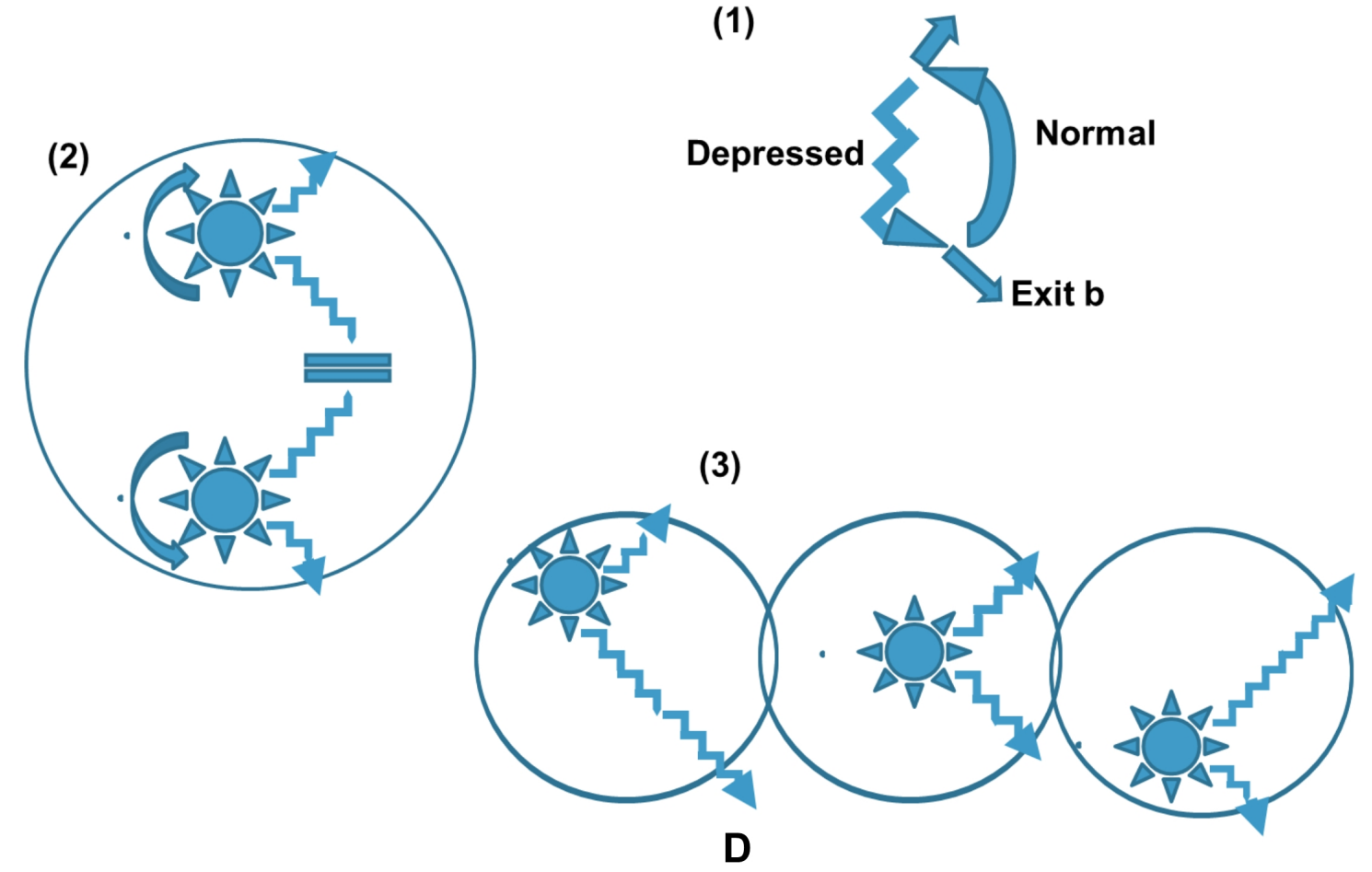

